\title{
DETERMINAÇÃo DOS PARÂMETROS DE ARRHENIUS DA REAÇÃo DE SORÇÃo DO DIÓXIDO DE ENXOFRE POR CALCÁRIO
}

\author{
Ivonete Ávila*, Paula M. Crnkovic e Fernando E. Milioli \\ Departamento de Engenharia Mecânica, Escola de Engenharia de São Carlos, Universidade de São Paulo, \\ Av. Trabalhador São-carlense, 400, 13560-970 São Carlos - SP, Brasil
}

Recebido em 16/10/06; aceito em 18/1/07; publicado na web em 2/7/07

\begin{abstract}
DETERMINATION OF THE ARRHENIUS PARAMETERS IN THE SULFUR DIOXIDE SORPTION REACTION BY LIMESTONE. Sulfur emission in coal power generation is a matter of great environmental concern and limestone sorbents are widely used for reducing such emissions. Thermogravimetry was applied to determine the effects of the type of limestone (calcite and dolomite), particle size (530 and $650 \mu \mathrm{m})$ and atmosphere (air and nitrogen) on the kinetics of $\mathrm{SO}_{2}$ sorption by limestone. Isothermal experiments were performed for different temperatures $\left(650\right.$ to $\left.950{ }^{\circ} \mathrm{C}\right)$, at local atmospheric pressure. The apparent activation energies, as indicated by the slope of the Arrhenius plot, resulted between 3.03 and $4.45 \mathrm{~kJ} \mathrm{~mol}^{-1}$ for the calcite, and $11.24 \mathrm{~kJ} \mathrm{~mol}^{-1}$ for the dolomite.
\end{abstract}

Keywords: sulfur dioxide; limestone; kinetics.

\section{INTRODUÇÃO}

A atmosfera terrestre pode ser dividida em camadas sobrepostas, conforme a variação da temperatura com a altitude. A camada mais baixa, onde vivemos e que está sujeita à ação poluente, é a troposfera que se estende desde a superfície até cerca de $15 \mathrm{~km}$ de altitude. Acima da troposfera encontra-se a estratosfera, que vai do topo da troposfera até aproximadamente $50 \mathrm{~km}$ da superfície da terra. Enquanto que na troposfera há um gradiente de temperatura que decresce com a altitude, na estratosfera, devido à absorção do ultravioleta pelo ozônio, há um gradiente de temperatura que cresce com a altitude e isto faz com que os poluentes gerados próximos ao solo fiquem confinados no interior da troposfera ${ }^{1,2}$.

Um fator importante na química troposférica é sua alta concentração de vapor de água, permitindo que poluentes, como o dióxido de enxofre $\left(\mathrm{SO}_{2}\right)$, possam reagir tanto em fase sólida (conversão homogênea) quanto em fase aquosa (conversão heterogênea). Vários fatores climatológicos interferem nestes processos, como umidade relativa e intensidade da radiação solar incidente ${ }^{3-5}$. Considerando-se a reação com o radical $\mathrm{OH}$, o tempo de vida médio do $\mathrm{SO}_{2}$ é de uma semana e, dependendo das condições de dispersão atmosféricas, pode atingir regiões distantes de dezenas a centenas de quilômetros ${ }^{2}$.

Atividades naturais e antropogênicas produzem os óxidos de enxofre $\left(\mathrm{SO}_{\mathrm{x}}\right)$. Entre as fontes naturais incluem-se vulcões, a decomposição de matéria orgânica e a vaporização do mar. Estas emissões aumentam a acidez da água da chuva, mas não chegam a causar danos ecológicos, pois o efeito do ácido é neutralizado ao entrar em contato com substâncias alcalinas presentes na água e no solo. O equilíbrio é rompido devido a grande quantidade de emissões de $\mathrm{SO}_{2}$ e isto ocorre especialmente nas regiões industrializadas do planeta, provocado pelas atividades humanas ${ }^{1,6}$.

A queima de combustíveis fósseis com elevados teores de enxofre para produção de energia é reconhecida como a principal fonte de emissão de $\mathrm{SO}_{2}$ na atmosfera. Entre os combustíveis poluidores de utilização em larga escala destacam-se os carvões minerais e os óleos pesados derivados do petróleo ${ }^{7}$.
O carvão mineral constitui-se em importante fonte de energia não renovável do Brasil, e apresenta-se como alternativa viável para suprir as crescentes demandas no setor energético brasileiro. Segundo o Ministério de Ciência e Tecnologia (MCT), o Brasil possui reservas de 31 bilhões de toneladas de carvão mineral e, atualmente, consome 5 milhões de toneladas por ano para geração de termoeletricidade. Isso corresponde a cerca de $2 \%$ da geração de energia elétrica no país. Em 2006 o MCT noticiou o investimento de $\mathrm{R}$ \$ 3,5 milhões em pesquisas, com o objetivo de aumentar para 5\% a participação do carvão mineral na geração de energia até o ano de $2015^{8}$.

Os problemas freqüentemente associados à queima dos carvões minerais referem-se principalmente à poluição ambiental. $\mathrm{O}$ carvão nacional varia, num sentido geral de sudeste para nordeste, de betuminoso de alto volátil $\mathrm{C}$ a betuminoso de alto volátil $\mathrm{A}$, pela classificação ASTM. Possui altos teores de cinzas, que variam de 40 a $60 \%$ e altos teores de enxofre (de 1 a $6 \%)^{9}$.

Considerável atenção tem sido focada à combustão de carvões minerais em reatores de leito fluidizado, que são de grande aplicação na queima de combustíveis potencialmente poluidores, principalmente visando a exploração termoelétrica ${ }^{7}$. O processo permite redução efetiva de emissões poluentes e consta entre a denominada tecnologia limpa do carvão ${ }^{1,10}$. Esta tecnologia permite a adição de calcários que, além do baixo custo também são eficientes sorventes de $\mathrm{SO}_{2}{ }^{11}$. Nestes reatores a combustão do carvão pode ocorrer entre 800 e $900{ }^{\circ} \mathrm{C}$ e isto é vantajoso, pois evita a formação de óxidos de nitrogênio $\left(\mathrm{NO}_{\mathrm{x}}\right)$ além de possibilitar a calcinação dos calcários. $\mathrm{O}$ processo de calcinação resulta na formação de óxido de cálcio $(\mathrm{CaO})$ e óxido de magnésio $(\mathrm{MgO})$, com liberação de dióxido de carbono $\left(\mathrm{CO}_{2}\right)$, afetando fisicamente o calcário que se torna mais poroso. A reação de calcinação de calcários, formados basicamente por carbonato de cálcio e de magnésio $\left(\mathrm{MCO}_{3}\right)$ é representada por:

$\mathrm{MCO}_{3}(\mathrm{~s}) \rightarrow \mathrm{MO}(\mathrm{s})+\mathrm{CO}_{2}(\mathrm{~g})$

Expostos ao $\mathrm{SO}_{2}$, os óxidos de cálcio e magnésio (MO) sulfatam produzindo principalmente sulfatos de cálcio e de magnésio $\left(\mathrm{MSO}_{4}\right)$ :

$\mathrm{MO}(\mathrm{s})+\mathrm{SO}_{2}(\mathrm{~g})+1 / 2 \mathrm{O}_{2}(\mathrm{~g}) \rightarrow \mathrm{MSO}_{4}(\mathrm{~g})$ 
Alguns autores afirmam que apenas o $\mathrm{CaO}$ dos calcários é sorvente de óxidos de enxofre $\left(\mathrm{SO}_{\mathrm{x}}\right)$ nas temperaturas de interesse em processos de combustão, posto que o produto $\mathrm{MgSO}_{4}$ se torna instável a altas temperaturas ${ }^{12,13}$. Outro argumento é que a reação do $\mathrm{MgO}$ com o $\mathrm{SO}_{2}$ é muito lenta e não ocorre sulfatação significativa ${ }^{14}$. Há também na literatura evidência da ocorrência de $\mathrm{MgSO}_{4}$ como produto da sulfatação ${ }^{15}$.

Em adição, resultados mais recentes obtidos no NETeF (Núcleo de Engenharia Térmica e Fluidos, - Departamento de Engenharia Mecânica, Escola de Engenharia de São Carlos, Universidade de São Paulo) apontam uma possível atividade do $\mathrm{MgO}^{16-18}$. Crnkovic et al. ${ }^{16}$ avaliaram a reatividade de $\mathrm{MgO}$ frente ao $\mathrm{SO}_{2}$ para estes mesmo calcários (calcítico e dolomítico) e nas mesmas condições experimentais deste estudo. Os autores concluíram que nos testes termogravimétricos, cuja concentração do $\mathrm{SO}_{2}$ foi de $20 \%$, deve-se considerar a fração de Mg presente na amostra como uma espécie reativa. Também utilizando os mesmos calcários, Camargo $^{17}$ estudou o processo de dessulfuração em um reator de leito fluidizado de bancada nas temperaturas de 750 e $850{ }^{\circ} \mathrm{C}$. Os resultados mostraram que, pelo balanço de massa, a conversão resultou em valores acima de $100 \%$, quando a massa de $\mathrm{Mg}$ foi excluída dos cálculos. Mesmos resultados também foram apresentados no trabalho de Pagliuso et al. ${ }^{18}$.

A identificação do mecanismo e do fator limitante das taxas de reação entre $\mathrm{CaO}$ e $\mathrm{SO}_{2}$ tem sido objeto de muitas pesquisas. No início da década de 70 , foram feitos os primeiros trabalhos demonstrando a influência da estrutura interna dos poros das partículas de $\mathrm{CaO}$ na reação com $\mathrm{SO}_{2}$. Com isso, passou-se a considerar aspectos físicos intrínsecos e não apenas efeitos superficiais externos, como até então considerados ${ }^{19}$. Em um dos primeiros trabalhos, verificou-se que partículas de calcário de $96 \mu \mathrm{m}$ impunham significativa resistência à difusão a $980{ }^{\circ} \mathrm{C}$. Os autores concluíram que a difusão de $\mathrm{SO}_{2}$ através das camadas do produto $\mathrm{CaSO}_{4}$ era o fator limitante da sulfatação ${ }^{20}$.

Vários trabalhos são relatados na literatura relacionados a estudos de sorção de $\mathrm{SO}_{2}$ por calcários em sistemas termogravimétricos ${ }^{10,11,16,21-26}$

Crnkovic ${ }^{11}$ estudou diferentes tipos de calcário brasileiros (calcítico, magnesiano e dolomítico) de diferentes procedências e diferentes idades geológicas (Itaú de Minas-MG, Ipeúna-SP e São José da Lapa-MG). Neste trabalho a autora utilizou amostras com faixas granulométricas estreitas entre 390 e $779 \mu \mathrm{m}$, em temperaturas de processo entre 600 e $900{ }^{\circ} \mathrm{C}$, em experimentos de calcinação seguida de sulfatação. Os resultados mostraram que o calcário mais jovem (dolomítico) mostrou-se mais reativo que o calcário mais antigo (calcítico). Este fato foi atribuído à geração de uma estrutura porosa mais acessível do calcário dolomítico em relação ao calcítico. Além disso, foi observado que há uma queda de reatividade para a reação de sulfatação em temperaturas acima de $850{ }^{\circ} \mathrm{C}$, devido possivelmente à sinterização de grãos de $\mathrm{CaO}$.

A cinética da reação de sulfatação foi estudada para o calcário dolomítico procedente de Ipeúna-SP, com granulometria média de $650 \mu \mathrm{m}^{11,16}$. Os resultados mostraram que a energia de ativação aparente $\left(E_{a}\right)$ variou com a temperatura, isto é, entre 600 e $700{ }^{\circ} \mathrm{C}$ a $E_{a}$ foi de $5,3 \mathrm{~kJ} \mathrm{~mol}^{-1}$; entre 700 e $850{ }^{\circ} \mathrm{C}$, foi de $11,6 \mathrm{~kJ} \mathrm{~mol}^{-1}$; entre 600 e $850{ }^{\circ} \mathrm{C}$, foi de $8,8 \mathrm{~kJ} \mathrm{~mol}^{-1}$. Os autores concluíram que as variações destes valores podem ser atribuídas às diferentes estruturas porosas em que o calcário se encontra, afetando de forma diferenciada o balanço entre as resistências à reação cinética $\mathrm{e}$ a difusão.

Alguns autores estudaram o processo de sulfatação em sistemas termogravimétricos, aplicando atmosfera com frações de $\mathrm{CO}_{2}$ altas o suficiente para inibir a calcinação do $\mathrm{CaCO}_{3}$. Sulfatação sob tais condições tem sido referida como processo de sulfatação $\operatorname{direta}^{10,13,21-23}$. Em alguns casos, altas concentrações de $\mathrm{CO}_{2}$ são usadas para simular o ambiente de combustor de leito fluidizado. Nestas condições a sulfatação direta do $\mathrm{CaCO}_{3}$ apresenta alta conversão, em contraste com a baixa sulfatação do calcário previamente calcinado.

Snow et $a l .{ }^{21}$ relataram estudos termogravimétricos nãoisotérmicos da sulfatação de $\mathrm{CaCO}_{3}$ para seis diferentes tamanhos de partículas, em atmosfera com diferentes concentrações de $\mathrm{CO}_{2}$ (de 2 a 95\%). Os autores encontraram energia de ativação de $64,1 \mathrm{~kJ} \mathrm{~mol}^{-1}$. Resultados semelhantes foram encontrados por Hajaligol et al. ${ }^{22}$, mesmo aplicando condições isotérmicas. Tanto Snow et al. ${ }^{21}$ quanto Hajaligol et al. ${ }^{22}$ concluíram que a difusividade depende da porosidade do sorvente. Estes trabalhos mostraram que em condições de sulfatação direta as camadas do produto sulfatado se tornam mais porosas, devido à liberação simultânea de $\mathrm{CO}_{2}$. A passagem de $\mathrm{CO}_{2}$ através da camada sulfatada leva a uma estrutura mais aberta, permitindo assim a um acesso mais fácil de $\mathrm{SO}_{2}$ e $\mathrm{O}_{2}$ para os sítios ativos das camadas de $\mathrm{CaO}$. Como conseqüência, a sulfatação direta torna-se mais efetiva comparada à sulfatação do calcário previamente calcinado.

Iisa e Hupa ${ }^{24}$ apresentaram um estudo cinético da reação de sulfatação empregando termogravimetria. Os autores utilizaram calcário com diâmetro médio entre 125 e $280 \mu \mathrm{m}$ e carbonato de cálcio com distribuição de diâmetros entre 1 e $5 \mu \mathrm{m}$. Entre 650 e $800{ }^{\circ} \mathrm{C}$, a energia de ativação aparente para o calcário resultou em $133 \mathrm{~kJ} \mathrm{~mol}^{-1} \mathrm{e}$, para o carbonato de cálcio resultou em $75 \mathrm{~kJ} \mathrm{~mol}^{-1}$ entre 875 e $950^{\circ} \mathrm{C}$. Os autores concluíram que para as partículas maiores, a sulfatação é limitada pela difusão através das camadas de produto, enquanto para as partículas menores, a sulfatação é controlada pela cinética da reação química.

Diferentes autores concordam que os altos valores relatados na literatura para energia de ativação aparente na sulfatação de calcário calcinado (entre 121 e $167 \mathrm{~kJ} \mathrm{~mol}^{-1}$ ) não são satisfatoriamente explicados, tendo em vista o controle difusivo de reação. Nesse caso resultariam energias de ativação em torno de $12 \mathrm{~kJ} \mathrm{~mol}^{-1}$ para controle por difusão molecular e $4 \mathrm{~kJ} \mathrm{~mol}^{-1}$ para controle por difusão de Knudsen ${ }^{20,27-29}$.

Este trabalho insere-se no contexto do programa de pesquisas em energia e poluição do NETeF. Uma das metas deste programa é contribuir para o uso dos carvões minerais brasileiros na geração termoelétrica, desenvolvendo tecnologia adequada para a redução de emissões poluentes. Neste trabalho, técnicas termogravimétricas foram empregadas com o objetivo de determinar a energia de ativação aparente no processo de sorção de $\mathrm{SO}_{2}$ por calcário, para diferentes condições de atmosfera, granulometria e tipo de calcário.

\section{PARTE EXPERIMENTAL}

Utilizaram-se dois tipos de calcário nacionais: calcítico procedente de Itaú de Minas-MG, denominado calcítico, com granulometrias médias de 545 e $650 \mu \mathrm{m}$, e dolomítico procedente de Ipeúna-SP, denominado dolomítico, com granulometria média de $650 \mu \mathrm{m}$. As granulometrias médias dos calcários foram obtidas por meio de seleção entre peneiras sucessivas da série ASTM, de modo a compor a faixa mais estreita possível e minimizar o efeito da distribuição granulométrica.

A composição química elementar dos calcários foi determinada por espectrometria de emissão atômica (ICP-AES). Os resultados da composição química dos calcários calcítico e dolomítico são apresentados na Tabela 1.

Os experimentos foram realizados em uma balança TG (Shimadzu - modelo TGA-51H). A massa de amostra empregada 
Tabela 1. Percentuais dos elementos encontrados nos calcários (dolomítico e calcítico)

\begin{tabular}{lccccccc}
\hline & $\mathrm{Ca}(\%)$ & $\mathrm{Mg}(\%)$ & $\mathrm{Fe}(\%)$ & $\mathrm{Al}(\%)$ & $\mathrm{Sr}(\%)$ & $\mathrm{K}(\%)$ & $\mathrm{Mn}(\%)$ \\
\hline Dolomítico & 17,07 & 11,73 & 0,32 & 0,42 & 0,08 & 0,13 & 0,09 \\
Calcítico & 35,34 & 0,35 & 2,45 & 0,25 & 0,14 & 0,09 & 0,52 \\
\hline
\end{tabular}

foi de aproximadamente $10 \mathrm{mg}$ de calcário em cada ensaio. Esta quantidade de massa garante que toda a amostra fique espalhada pelo suporte em uma monocamada, minimizando as resistências da transferência de massa nos interstícios entre partículas.

O suporte de amostra (ou do cadinho) utilizado foi de alumina com 5,8 $\mathrm{mm}$ de diâmetro e 2,6 $\mathrm{mm}$ de altura. A escolha do cadinho de alumina foi feita pelo fato deste material suportar altas temperaturas e não apresentar efeitos catalíticos sobre a reação de sulfatação quando comparado ao cadinho de platina ${ }^{11,30}$.

A amostra (calcário natural) foi colocada no cadinho e este introduzido no forno da balança TG. Inicialmente, foi feita a calcinação da amostra em atmosfera dinâmica de ar sintético ou de nitrogênio $\left(80 \mathrm{~mL} \mathrm{~min}^{-1}\right)$, com razão de aquecimento de $30{ }^{\circ} \mathrm{C} \mathrm{min}{ }^{-1}$ até a temperatura desejada. Após a calcinação e 5 min de estabilização, o gás $\mathrm{SO}_{2}$ foi injetado no forno da balança sob condição isotérmica durante $20 \mathrm{~min}$ com vazão de $20 \mathrm{~mL} \mathrm{~min}^{-1}$. A fração de $\mathrm{SO}_{2}$ ficou em $20 \%$ da atmosfera total. A alta concentração de $\mathrm{SO}_{2}$ no meio reacional confere ao sistema a condição denominada "condição diferencial", em que se elimina o efeito de resistência do transporte de massa externa do gás reagente $\left(\mathrm{SO}_{2}\right)$ até a camada mais externa da partícula ${ }^{15,16}$

Foram consideradas diferentes temperaturas nos ensaios isotérmicos de sulfatação (entre 650 e $950{ }^{\circ} \mathrm{C}$ ) e para cada ensaio foram feitas quatro repetições. Nos ensaios isotérmicos em temperaturas abaixo de $850{ }^{\circ} \mathrm{C}$, previamente à sulfatação, foi necessário levar a temperatura até $850{ }^{\circ} \mathrm{C}$ para promover a calcinação completa da amostra. Nestes experimentos a razão de aquecimento foi de $30{ }^{\circ} \mathrm{C} \mathrm{min}{ }^{-1}$ até $850{ }^{\circ} \mathrm{C}$, permanecendo nesta temperatura por $5 \mathrm{~min}$, quando então se iniciou o resfriamento com razão de $30{ }^{\circ} \mathrm{C} \mathrm{min}^{-1}$ até a temperatura desejada. Em seguida, procedeu-se conforme descrito anteriormente.

Para os testes de porosimetria de mercúrio, necessita-se de amostras de calcários com massa aproximada de $500 \mathrm{mg}$. As amostras foram preparadas em forno tubular com volume de $3.817 \mathrm{~cm}^{3}$, com aquecimento controlado de modo a se obter as mesmas condições de razão de aquecimento e isotermas feitas em balança termogravimétrica.

As curvas por intrusão de mercúrio foram obtidas em porosímetro Micrometrics Pore Size 9320 e as imagens da estrutura morfológica foram feitas em microscópio eletrônico de varredura (MEV) Leo Electron Microscopy 440 - Zeiz/Laika.

O coeficiente de taxa ou constante de velocidade de reação $(k)$ é modelado aplicando-se a expressão de Arrhenius, ou seja:

$k(T)=A \exp \left(-\frac{E_{a}}{R T}\right)$

sendo $A$ o fator pré-exponencial ou fator de frequiência; $E_{a}$ a energia de ativação aparente; $R$ a constante universal dos gases e $T$ a temperatura.

Em processos reativos gás-sólido, os resultados cinéticos incorporam difusão gasosa intrapartícula, difusão de Knudsen e difusão iônica. Assim, para estes casos, é comum na literatura a denominação de energia de ativação aparente e de ordem de reação aparente $^{11}$.
Na Equação 3 o termo pré-exponencial, conhecido por fator ou distribuição de Boltzmann, especifica a fração das colisões com energia superior à energia de ativação. Multiplicado pela fração de colisões efetivas ou favoráveis, representada pelo fator préexponencial, define-se a frequiência de reação ou coeficiente de taxa. A taxa de reação de sorção pode ser dada por:

$-\frac{d m}{d t} \propto \mathrm{m}^{\mathrm{a}} \mathrm{C}_{\mathrm{SO}_{2}}^{\mathrm{b}} \mathrm{C}_{\mathrm{O}_{2}}^{\mathrm{c}}$

sendo $d m / d t$ a taxa de reação de Ca e Mg disponíveis na amostra de calcário, $m$ as respectivas massas disponíveis para a reação, $C$ a concentração dos gases na atmosfera da balança e os índices $(a, b$, c) a ordem da reação global em relação à massa ou a cada um dos gases reagentes.

Considera-se reação de primeira ordem em relação à massa da amostra e independente das concentrações de gases reagentes, isto é, assume-se pseudo-ordem zero em relação às concentrações dos gases reagentes. Na literatura não há informação em relação à ordem de reação do processo de sulfatação por calcários e, em geral, assume-se ordem 1. Quanto aos gases reagentes, aplica-se concentrações altas o suficiente para que seus valores não afetem as taxas de reação (20\% para $\mathrm{SO}_{2}$ e $16 \%$ para $\mathrm{O}_{2}$ ). Então tem-se:

$-\frac{d m}{d t} \propto \mathrm{m}$

Introduzindo-se um coeficiente de taxa de reação $(k)$, da Equação 5 tem-se:

$-\frac{d m}{d t}=k m$

Substituindo-se a Equação 6 na Equação 3 e aplicando o operador logaritmo natural, obtém-se:

$\ln \left[-\frac{1}{m} \frac{d m}{d t}\right]=\ln A-\frac{E_{a}}{R} \frac{1}{T}$

Os dados obtidos experimentalmente a partir das curvas TG e DTG estão relacionados com os valores da amostra de calcário. Neste estudo, a massa transiente $(m)$ corresponde à fração de cálcio e magnésio disponíveis no calcário para a reação. O valor de $m$ é obtido do balanço de massa, assumindo-se que $\mathrm{Ca}$ e $\mathrm{Mg}$ sejam consumidos à mesma taxa de reação. Em atmosfera de ar sintético $m$ resulta em:

$m=M_{A}\left(Y_{C a}+Y_{M g}\right)-\left\{\left(M-M_{c}\right)\left[\frac{\left(W_{C a} Y_{C a}+W_{M g} Y_{M g}\right)}{\left(W_{S O_{2}}+1 / 2 W_{O_{2}}\right)\left(Y_{C a}+Y_{M g}\right)}\right]\right\}$

sendo $M_{\mathrm{A}}$ a massa inicial da amostra (calcário natural); $M$ a massa transiente da amostra durante a sulfatação; $M_{C}$ a massa do calcário calcinado; $Y$ a fração em massa de $\mathrm{Ca}$ e $\mathrm{Mg}$ no calcário natural (Tabela 1$) ; W$ a massa molar de cada um dos componentes $(\mathrm{Ca}$, $\mathrm{Mg}, \mathrm{SO}_{2}$ e $\mathrm{O}_{2}$ ).

Deriva-se então a Equação 8 em relação ao tempo e obtém-se:

$\frac{d m}{d t}=-\left[\frac{\left(W_{C a} Y_{C a}+W_{M g} Y_{M g}\right)}{\left(W_{S_{2}}+1 / 2 W_{O_{2}}\right)\left(Y_{C a}+Y_{M g}\right)}\right] \frac{d M}{d t}$

Em atmosfera de nitrogênio o termo $1 / 2 W_{O_{2}}$ é excluído das Equações 8 e 9.

Os valores de $M_{A}, M_{C}$ e $M$ são obtidos na curva TG, dados em mg, 
e os valores de $d M / d t$ são obtidos na curva DTG, dados em $\mathrm{mg} \mathrm{s}^{-1}$, dos experimentos termogravimétricos.

A partir dos dados empíricos e das Equações 8 e 9 calculam-se os valores do primeiro termo à esquerda da Equação 7 em cada temperatura estudada. Representa-se, então, graficamente $\ln \left[\frac{1}{m} \frac{d m}{d t}\right]$ versus $1 / T(\mathrm{~K})$. Pelo ajuste linear obtém-se o coeficiente angular $\left(-E_{a} / R\right)$ e o coeficiente linear $(\ln A)$ da reta.

\section{RESULTADOS E DISCUSSÃO}

A Figura 1 mostra as curvas obtidas na calcinação seguida de sulfatação para um dos ensaios termogravimétricos realizados. São mostrados os perfis TG, DTG e razão de aquecimento $\left(30^{\circ} \mathrm{C} \mathrm{min}^{-1}\right)$. Os indicadores [1] e [2] sobre a curva TG indicam os patamares entre os quais se desenvolve a perda de massa devido à calcinação. Após a calcinação completa da amostra, $\mathrm{SO}_{2}$ é injetado na atmosfera do forno, promovendo-se a reação de sulfatação. O ganho de massa na sulfatação ocorre entre os patamares [2] e [3].

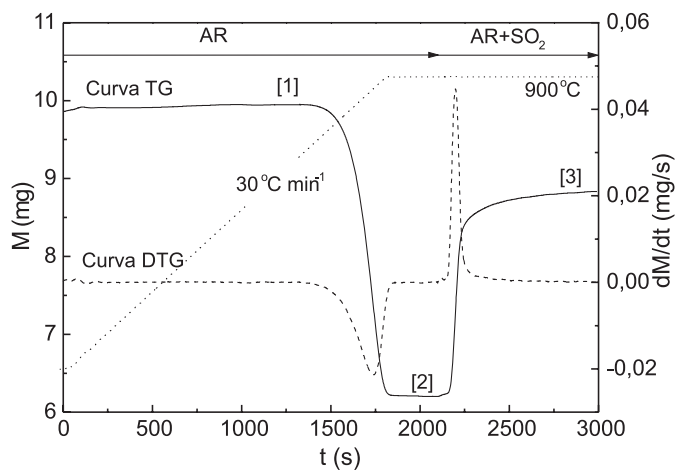

Figura 1. Curvas TG e DTG em função do tempo (t) do processo de calcinação seguido de sulfatação para um ensaio a $900{ }^{\circ} \mathrm{C}$ em atmosfera dinâmica de ar sintético

Foram feitos quatro ensaios termogravimétricos para cada temperatura ensaiada $(750,800,850$ e 900$)$ em cada atmosfera estudada (ar sintético e nitrogênio). A Figura 2 apresenta um conjunto destes testes para a temperatura de $900{ }^{\circ} \mathrm{C} \mathrm{em}$ atmosfera de ar sintético. Nota-se que em todas as curvas TG, apresentadas na Figura 2, há uma perda de massa entre o tempo de 1200 e 1800 s correspondente à liberação de $\mathrm{CO}_{2}$ e um ganho de massa entre o tempo de 2100 e $3000 \mathrm{~s}$, correspondente à sulfatação.

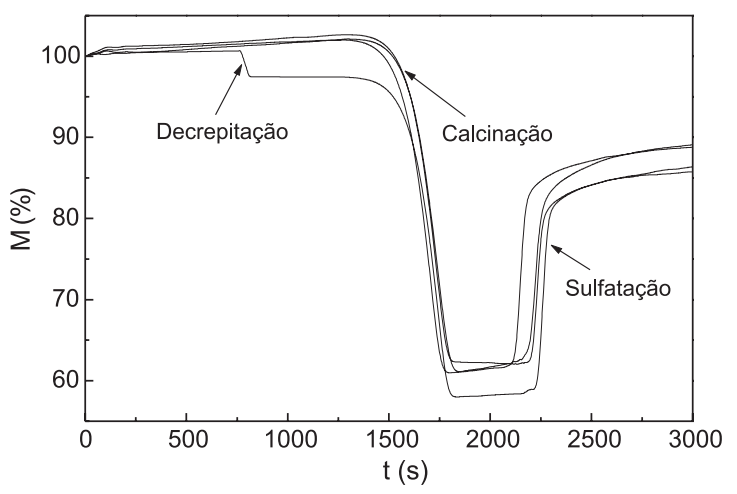

Figura 2. Conjunto de curvas TG em função do tempo (t) de quatro ensaios do processo de calcinação seguido de sulfatação na temperatura de $900{ }^{\circ} \mathrm{C}$ em atmosfera de ar sintético para o calcário calcítico com granulometria de $545 \mu \mathrm{m}$. Observa-se o fenômeno da decrepitação em uma das curvas
Observa-se na Figura 2 que, em um dos ensaios, antes do processo de calcinação, ocorreu uma perda brusca de massa em torno de $700 \mathrm{~s}$ atribuída à decrepitação. Este é um fenômeno termomecânico provocado por pequenas explosões dentro das partículas, devido à pressão gerada pela evaporação da água no retículo cristalino. Quando ocorre este evento no ensaio TG, as explosões podem fazer com que algumas partículas sejam lançadas para fora do suporte da amostra, indicando nas curvas TG uma perda de massa ${ }^{31,32}$. A decrepitação ocorreu em alguns dos experimentos com o calcário calcítico, nas duas atmosferas e nas duas granulometrias ensaiadas, enquanto que não ocorreu em nenhum dos experimentos com o calcário dolomítico. É importante observar que este fenômeno não interfere nos resultados, pois para os cálculos utiliza-se como valor da massa inicial da amostra a massa imediatamente anterior ao início da calcinação. Também na Figura 2 observa-se que, nestas condições estudadas, em média $38 \%$ da massa inicial calcinou e desta, em média $30 \%$ formou o produto sulfatado.

Na Figura 3 estão representadas as curvas da derivada temporal, ou curvas DTG, referentes às curvas TG apresentadas na Figura 2. Nota-se picos DTG referentes ao fenômeno da decrepitação em um dos testes (em torno $700 \mathrm{~s}$ ), a perda de massa correspondente à calcinação (em torno de $1500 \mathrm{~s}$ ) e ao ganho de massa no processo de sulfatação (em torno de 2100 s).

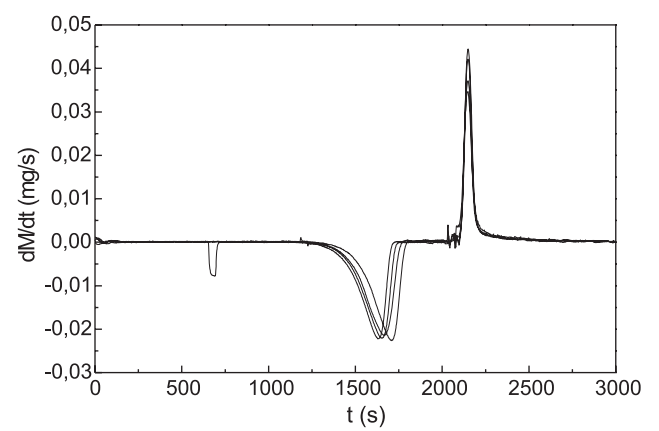

Figura 3. Conjunto de curvas DTG em função do tempo (t) obtidas das curvas TG da Figura 2, para os quatro ensaios do processo de calcinação seguido de sulfatação na temperatura de $900^{\circ} \mathrm{C}$ em atmosfera de ar sintético

Foram obtidas as curvas TG e DTG para todas as condições estudadas como já descrito e é a partir do pico da curva DTG referente à reação de sulfatação (após 2000 s) que se obtém o valor de dM/dt para ser utilizado na Equação 9. Neste caso, os parâmetros de Arrhenius foram determinados no ponto onde a taxa de reação é máxima.

Determina-se assim um coeficiente de taxa de reação máxima $\left(K_{\max }\right)$ e a Equação 9 passa a ser escrita como:

$\ln \left[-\left(\frac{1}{m} \frac{d m}{d t}\right)_{\max }\right]=\ln A-\frac{E_{a}}{R} \frac{1}{T}$

A representação gráfica do conjunto de pontos obtidos em função da temperatura fornece os parâmetros cinéticos de Arrhenius pelo ajuste da reta. Os valores do fator pré-exponencial $A\left(\mathrm{~s}^{-1}\right)$ e da energia de ativação aparente $E_{a}\left(\mathrm{~kJ} \mathrm{~mol}^{-1}\right)$ foram obtidos, respectivamente pelo coeficiente linear e coeficiente angular. A Figura 4 apresenta estes resultados .

Na Figura 4 notam-se três pontos à esquerda, que não pertencem às retas obtidas nos testes em atmosfera de ar sintético. Inicialmente estava previsto obter os parâmetros de Arrhenius entre as temperaturas de 750 e $950{ }^{\circ} \mathrm{C}$ para ambas as atmosferas estudadas (ar sintético e nitrogênio). Entretanto, em atmosfera de ar sintético, as taxas de reação médias resultantes nas temperaturas de 900 
e $950{ }^{\circ} \mathrm{C}$ para o calcário calcítico com granulometria de $545 \mu \mathrm{m}$ foram inferiores às taxas obtidas nas temperaturas de 750 a $850{ }^{\circ} \mathrm{C}$. Este fato pode ser atribuído à mudança nas condições de reação nas temperaturas mais elevadas, possivelmente devido à sinterização do sorvente. Este mesmo comportamento foi também observado para os calcários calcítico e dolomítico com granulometria de $650 \mu \mathrm{m}$ em atmosfera de ar sintético. Nestes casos, escolheu-se a faixa de temperaturas de 650 a $850{ }^{\circ} \mathrm{C}$ para se determinar os parâmetros cinéticos para a atmosfera de ar sintético. Assim, em atmosfera de ar sintético excluíram-se os pontos acima de $850{ }^{\circ} \mathrm{C}$ e aplicou-se a análise a partir de 5 pontos (de 650 a $850{ }^{\circ} \mathrm{C}$ ), mantendo-se a mesma qualidade na caracterização do comportamento cinético como previsto inicialmente.

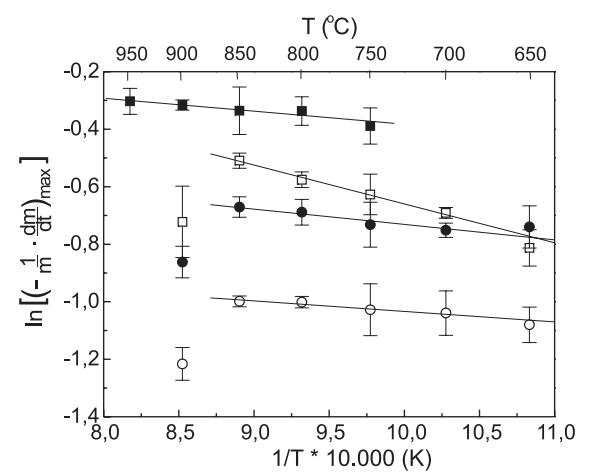

Figura 4. Coeficiente de taxa de reação no instante de máxima taxa de reação $\left(\mathrm{k}_{\max }\right)$ em função da temperatura $(\mathrm{T})$ para as condições estudadas, sendo: - Calcítico $545 \mu \mathrm{m} \mathrm{N}$; $\square$ - Dolomítico 650 um em ar sintético; - Calcítico $545 \mu \mathrm{m}$ em ar sintético e $\bigcirc$ - Calcítico $650 \mu \mathrm{m}$ em ar sintético. As barras indicam o desvio padrão em torno da média

Nos ensaios em atmosfera de nitrogênio não foi observada mudança nas condições de reação nas temperaturas mais elevadas $\left(900\right.$ e $950{ }^{\circ} \mathrm{C}$ ). Devido a isto, os parâmetros cinéticos foram determinados como inicialmente previsto, ou seja, entre as temperaturas de 750 e $9500^{\circ} \mathrm{C}$.

Na Tabela 2 apresentam-se os fatores pré-exponenciais, energias de ativação e os coeficientes de taxa de reação máxima a $850{ }^{\circ} \mathrm{C}\left(k_{\max }\right)$, obtidos entre as temperaturas de 650 e $850{ }^{\circ} \mathrm{C}$, para atmosfera de ar sintético, e entre as temperaturas de 750 a $950{ }^{\circ} \mathrm{C}$ para atmosfera de $\mathrm{N}_{2}$.

Em atmosfera de ar sintético, o calcário dolomítico apresentou um fator de freqüência aproximadamente 3,6 vezes mais alto que aquele obtido para o calcário calcítico de mesma granulometria. Este resultado mostra que o calcário dolomítico é 3,6 vezes mais efetivo que o calcário calcítico. Por outro lado, a energia de ativação aparente para o calcário dolomítico resultou aproximadamente 3,7 vezes maior que a energia de ativação aparente obtida para o calcário calcítico. Isto mostra que o calcário dolomítico requer 3,7 vezes mais energia para que a reação ocorra.

Comparando-se as duas granulometria estudadas (545 e $650 \mu \mathrm{m})$ para o calcário calcítico em atmosfera de ar sintético, observa-se que tanto o fator de frequiência quanto a energia de ativação aparente aumentaram com a diminuição da granulometria. Em granulometria menor a reação foi mais efetiva, pois ocorreu aumento de colisões, evidenciado pelo aumento do fator de frequiência. Este fato pode ser atribuído à maior área superficial das partículas menores. Entretanto, esperava-se que a energia de ativação permanecesse a mesma ou muito próxima para as duas granulometrias, uma vez que se trata do mesmo calcário. Esta diferença pode ser atribuída à heterogeneidade da amostra e partículas com formas diferentes e não esféricas. Notase também que a diferença entre as duas granulometrias estudadas não é muito grande (em torno de $20 \%$ ).

A mudança da atmosfera de ar sintético para nitrogênio, para o calcário calcítico com granulometria de $545 \mu \mathrm{m}$, resultou no aumento do fator de frequiência e na diminuição da energia de ativação aparente. Este resultado mostra que em atmosfera de nitrogênio a reação de sulfatação é mais efetiva e requer menor energia para ocorrer. É importante ressaltar que a avaliação dos parâmetros cinéticos foi feita em faixas de temperaturas diferentes para a atmosfera de ar sintético (entre 650 a $850{ }^{\circ} \mathrm{C}$ ) e de nitrogênio (entre 750 a $950^{\circ} \mathrm{C}$ ). Porém, notase que há um intervalo em comum (de 750 a $850{ }^{\circ} \mathrm{C}$ ) tornando comparáveis os resultados obtidos nas atmosferas estudadas.

Para o calcário calcítico a energia de ativação resultou entre 3,03 e 4,45 kJ mol ${ }^{-1}$, sugerindo processo reativo controlado por difusão de Knudsen ${ }^{20,27-29}$. No caso do calcário dolomítico a energia de ativação resultou em 11,24 $\mathrm{kJ} \mathrm{mol}^{-1}$, sugerindo processo reativo controlado por difusão molecular do gás através dos poros ${ }^{20,27-29}$.

Os parâmetros cinéticos obtidos na reação de sulfatação de calcário são de extrema importância no dimensionamento de reatores quando se utilizam sorventes calcários em processos de combustão de carvão mineral em leito fluidizado. A fim de avaliar a capacidade de sorção dos calcários, calculou-se a conversão no processo de sulfatação a $850{ }^{\circ} \mathrm{C}$, temperatura típica do processo de combustão em leito fluidizado. Em processo de regime estacionário, a quantidade de mol da espécie química $i$ que reage é igual à diferença entre a quantidade de mols de $i$ que entra no processo $\left(n_{e}\right)$ e a quantidade de mols de $i$ que sai $\left(n_{s}\right)$. Desta forma, a conversão $(X)$ na forma percentual é dada por $^{32}$ :

$$
X=\frac{\frac{M-M_{C}}{W_{s o_{2}}+1 / 2 W_{O_{2}}}}{M_{A}\left(\frac{Y_{C a}}{W_{C a}}+\frac{Y_{M g}}{W_{M g}}\right)} \times 100
$$

A conversão final do calcário é obtida quanto $M=M_{F}$, sendo $M_{F}$ a massa final após a sulfatação. Nesse caso considera-se o tempo inicial $(t=0) 100 \mathrm{~s}$ antes do pico da DTG e o tempo final igual a $400 \mathrm{~s}$ após o tempo inicial. Assim como no estudo cinético, em atmosfera de nitrogênio o termo 1/2 Woz também foi excluído da Equação 11.

Os resultados de conversão transiente a $850{ }^{\circ} \mathrm{C}$ são apresentados na Figura 5. As curvas de conversão permitem quantificar a conversão final, ou reatividade final, dos sorventes e os valores estão apresentados na Tabela 3. Nota-se que a conversão é fortemente afetada pela atmosfera, granulometria e tipo de calcário. Observa-se que o calcário dolomítico apresenta maior reatividade quando comparada à do calcítico. Segundo Milne et al..$^{33}$ a reação de $\mathrm{SO}_{2}$ com $\mathrm{CaO}$ é inicialmente rápida e então limitada por proces-

Tabela 2. Parâmetros de Arrhenius obtidos da reação de sorção do dióxido de enxofre pelos calcários (dolomítico e calcítico)

\begin{tabular}{llccccc}
\hline & Atmosfera & Granulometria $[\mathrm{m}]$ & Faixa de Temperatura $\left[{ }^{\circ} \mathrm{C}\right]$ & $A\left[\mathrm{~s}^{-1}\right]$ & $E_{a}\left[\mathrm{~kJ} \mathrm{~mol}^{-1}\right]$ & $k_{\max }\left[\mathrm{s}^{-1}\right]$ \\
\hline Dolomítico & ar sintético & 650 & $650-850$ & 1,997 & 11,24 & 0,60 \\
Calcítico & ar sintético & 650 & $650-850$ & 0,5512 & 3,03 & 0,40 \\
& ar sintético & 545 & $650-850$ & 0,822 & 4,45 & 0,51 \\
& nitrogênio & 545 & $750-950$ & 1,071 & 3,75 & 0,72 \\
\hline
\end{tabular}


sos difusionais, que são fortemente dependentes da estrutura da partícula. Com base nos presentes dados, supõe-se que as estruturas físicas sejam diferentes.

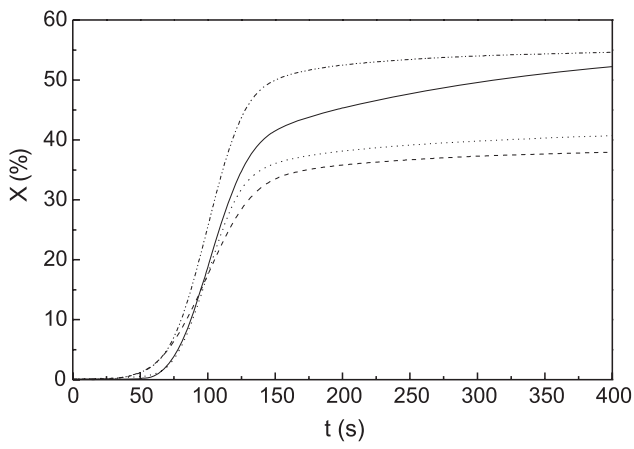

Figura 5. Conversão média (X) em função do tempo (t) do processo de sulfatação a $850^{\circ} \mathrm{C}$, sendo: ---Calcítico $545 \mu \mathrm{m} \mathrm{N}_{2}$; -Dolomítico 650 um em ar sintético; --Calcítico 545 um em ar sintético; --Calcítico 545 ìm em ar sintético

Esta suposição pode ser comprovada através das imagens obtidas por Microscopia Eletrônica de Varredura (MEV) apresentadas nas Figuras 6 e 7. Observa-se que as estruturas morfológicas dos calcários apresentam-se diferentes quanto à compactação.

A Figura 6 mostra as imagens com ampliação de 5000 vezes dos calcários na forma natural. Nota-se que o calcário dolomítico apresenta uma estrutura mais porosa em relação ao calcário calcítico. Na Figura 7 apresentam-se as imagens, também com ampliação de 5000 vezes, para os calcários calcinados. Observa-se que a estrutura morfológica do calcário dolomítico se manteve após a calcinação. Já no calcário calcítico, que quando natural apresenta uma estrutura mais compacta, após a calcinação nota-se uma estrutura superficial mais porosa, semelhante ao calcário dolomítico.

Embora estas imagens permitam observar a diferença quanto à porosidade, esta avaliação é apenas qualitativa. Para uma determinação quantitativa, utilizou-se a porosimetria de mercúrio para ambos os calcários, tanto na forma natural quanto calcinada. Os resultados são apresentados na Tabela 3. Nota-se que após a calcinação houve um aumento de porosidade de $79 \%$ para o calcário calcítico e de $45 \%$ para o dolomítico.

Tabela 3. Porosidade dos calcários (dolomítico e calcítico) na forma natural e calcinado

\begin{tabular}{lccc}
\hline & \multicolumn{2}{c}{ Porosidade (\%) } & Conversão (\%) \\
& Natural & Calcinado & \\
\hline Calcítico & 15,12 & 27,11 & $52,1 \pm 1,4$ \\
Dolomítico & 19,70 & 28,52 & $38,0 \pm 0,7$ \\
\hline
\end{tabular}

A energia de ativação determinada para o calcário calcítico $\left(\sim 4 \mathrm{~kJ} \mathrm{~mol}^{-1}\right)$ mostra que a sua sulfatação é controlada por difusão de Knudsen ${ }^{20,27-29}$. A difusão de Knudsen ocorre quando o livre caminho médio entre moléculas do gás é maior que o diâmetro de poros do sólido. Neste caso, as moléculas colidem mais frequientemente com as paredes dos poros que entre $\mathrm{si}^{34}$, o que pode justificar os menores valores de energia de ativação, quando comparado com o calcário dolomítico. Isto é indicativo que a estrutura morfológica do calcário calcítico é caracterizada por poros com diâmetros médios pequenos, levando mais rapidamente ao bloqueio dos mesmos, impondo baixa conversão no processo de sulfatação.

Em contrapartida, valores de energia de ativação da ordem obtida para o calcário dolomítico $\left(\sim 12 \mathrm{~kJ} \mathrm{~mol}^{-1}\right)$ correspondem a um mecanismo satisfatoriamente explicado considerando a difusão

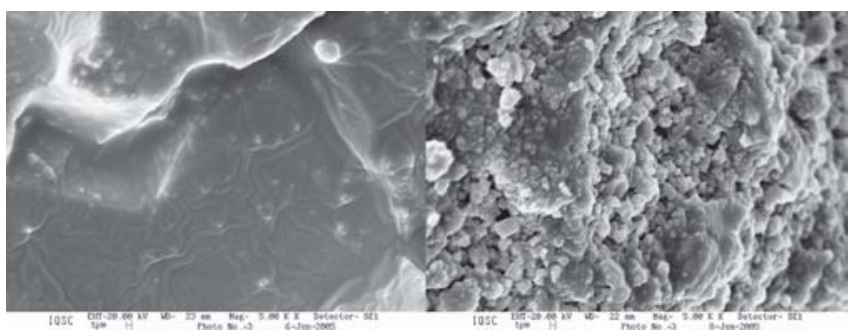

(a)

(b)

Figura 6. Microscopia Eletrônica de Varredura (MEV) com ampliação de 5000x do calcário natural: (a) Calcítico; (b) Dolomítico

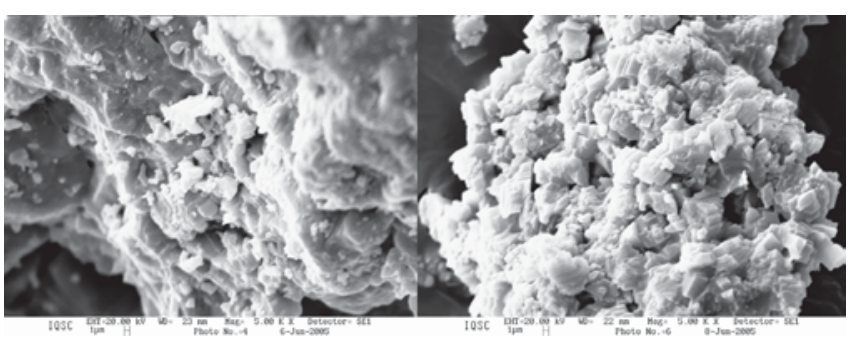

(a)

(b)

Figura 7. Microscopia Eletrônica de Varredura (MEV) com ampliação de 5000x do calcário calcinado: (a) Calcítico; (b) Dolomítico

molecular ${ }^{20,27-29}$. Neste processo, o reagente se difunde nos poros do sólido, sendo que a concentração deste na entrada do poro é maior que no seu interior ${ }^{31}$. Isto é indicativo de que, apesar da dificuldade de acesso aos poros mais internos, quando esta barreira é ultrapassada, maiores conversões serão obtidas para a estrutura interna mais porosa e com poros de maior diâmetro médio. Os resultados indicam que a estrutura morfológica interna do calcário dolomítico é mais porosa e com diâmetro médio maior quando comparada à do calcítico. Tal porosidade permite maiores conversões antes que se atinja o bloqueio de poros. A conversão final do calcário dolomítico resultou cerca de $14 \%$ superior à do calcário calcítico.

A diferença observada para os dois tipos de calcários estudados (calcítico e dolomítico) sugere que a estrutura física é um fator importante na reatividade do sorvente no processo de sulfatação, afetando diretamente a cinética da reação.

\section{CONCLUSÕES}

Os valores de energia de ativação relatados na literatura variam desde unidades até centenas de $\mathrm{kJ} \mathrm{mol}^{-1}{ }^{13,21,23}$. Essas diferenças se devem a vários fatores, incluindo tipo do calcário, idade geológica ${ }^{26}$, composição química, heterogeneidade dos calcários ${ }^{35}$, porosidade, tamanho e distribuição de poros, área superficial interna ${ }^{7,19,36}$, além das condições de ensaios, como atmosfera, pressão, entre outras.

A análise cinética mostrou que, em comparação com o calcário calcítico, o calcário dolomítico apresentou maiores valores para o fator de freqüência e a energia de ativação aparente.

O calcário calcítico com tamanho de partícula menor, comparando-se as granulometrias de 545 e $650 \mu \mathrm{m}$, apresentou maiores valores de fator de freqüência e da energia de ativação aparente. Também para este calcário, a mudança da atmosfera de ar sintético para nitrogênio causou aumento no valor do fator de frequiência e diminuição da energia de ativação aparente.

Considerando-se a ordem de magnitude dos valores da energia de ativação aparente, concluiu-se que o processo de sulfatação para calcário calcítico é controlado por difusão de Knudsen, enquanto que para o calcário dolomítico o processo reativo é controlado por 
difusão molecular do gás através dos poros.

A estrutura morfológica dos calcários estudados apresentou diferenças de compactação na forma natural, sendo que o calcário calcítico possui uma estrutura menos porosa que o calcário dolomítico. Após a calcinação houve um aumento de porosidade de $79 \%$ para o calcário calcítico e de $45 \%$ para o dolomítico.

Os resultados mostraram maiores valores de conversão no processo de sulfatação para o calcário dolomítico, indicando que a estrutura morfológica é um fator importante na reatividade do sorvente.

Novos estudos estão programados para serem realizados no $\mathrm{NETeF}$, onde se pretende cumprir um extenso programa experimental para correlacionar a reatividade com porosidade, distribuição de tamanhos de poros e área superficial BET.

\section{AGRADECIMENTOS}

Este trabalho teve suporte financeiro da Fundação de Amparo à Pesquisa do Estado de São Paulo - FAPESP (Processos 04/068944 e $05 / 50152-5$ ).

\section{REFERÊNCIAS}

1. Baird, C.; Environmental Chemistry, $2^{\text {nd }}$ ed., W.H. Freeman and Company: New York, 1998.

2. Seinfeld, J.; Pandis, S.; Atmospheric Chemistry and Physics - From Air Pollution to Climate Change, John Wiley \& Sons: New York, 1998.

3. Cardoso, A. A.; Pitombo, L. R. M.; Quim. Nova 1992, 15, 219.

4. Derisio, J. C.; Introdução ao Controle de Poluição Ambiental, $1^{\mathrm{a}}$ ed., Cetesb: São Paulo, 1992.

5. Wark, K.; Warner, C. F.; Davis, W. T.; Air pollution: Its origin and control, $3^{\text {rd }}$ ed., Manlo Park: California: Addisonj-Wesley, 1998.

6. Kotz, J. C.; Joesten, M. D.; Wood, J. L.; Moore, J. W.; The Chemical World: Concepts and Applications, Harcourt Brace \& Company: Orlando, 1994

7. Li, Y.; Sadakata, M.; Fuel 1999, 78, 1089.

8. http://www.mct.gov.br/index.php/content/view/5079.html, acessada em Agosto 2006.
9. Süffert, T.; Carvão nos Estados do Rio Grande do Sul e Santa Catarina, Informe de Recursos Minerais, Série Oportunidades Minerais - Exame Atualizado de Projetos - n. ${ }^{\circ}$ 21, CPRM: Porto Alegre, 1997.

10. Van Houte, G.; Delmon, B. ; J. Chem. Soc. Faraday Trans. I 1979, 75, 1593.

11. Crnkovic, P. C. G. M.; Tese de Doutorado, Universidade de São Paulo, Brasil, 2003.

12. Wieczorek-Ciurowa, K.; J. Therm. Anal. Calorim. 1992, 38, 523.

13. Fuertes, A. B.; Velasco, G., Fuente, E.; Alvarez, T.; Fuel Process. Technol. 1994, 38, 181.

14. Borgwardt, R. H.; Harvey, R. D.; Environ. Sci. Technol. 1972, 6, 350

15. Borgwardt, R. H.; Environ. Sci. Technol. 1970, 4, 59.

16. Crnkovic, P. M.; Milioli, F. E.; Pagliuso, J. D.; Thermochim. Acta 2006, 447, 161.

17. Camargo, F. L.; Dissertação de Mestrado, Universidade de São Paulo, Brasil, 2001.

18. Pagliuso, J. D.; Milioli, F. E.; Crnkovic, P. M.; Costa, M. C. D., Silva, G. F. Camargo, F. L.; AIChE Annual Meeting, Indianapolis, USA, 2002.

19. Borgwardt, R. H.; Bruce, K. R.; AIChE J. 1986, 32, 239.

20. Pigford, R.; Sliger, G.; Ind. Eng. Chem. Process Des. Develop. 1973, 12, 85

21. Show, M. J. H.; Longwell, J. P.; Sarofim, A. F.; Ind. Eng. Res. 1988, 27,

22. Hajaligol, M. R.; Longwell, J. P.; Sarofim, A. F.; Ind. Eng. Res. 1988, 27, 2203.

23. Zhong, Q.; Thermochim. Acta 1995, 260, 125.

24. Iisa, K.; Hupa, M.; J. Inst. Energy 1992, 65, 201.

25. Adánez, J.; Garciá-Labiano, F.; Gayán, P.; Fuel Process. Technol. 1993, 36,73

26. Yrjas, P.; Iisa, K.; Hupa, M.; Fuel 1995, 74, 395

27. Bhatia, S. K.; Perlmutter, D. D.; AIChE J. 1981, 27, 226.

28. Gopalakrishanan, R.; Seehra, M. S.; Energy Fuels 1990, 4, 226.

29. Fuertes, A.B.; Fernandez, M. J.; Trans. IChemE 1995, 73, 854.

30. Wieczorek-Ciurowa, K.; J. Therm. Anal. 1992, 38, 2101.

31. Crnkovic, P. M.; Polito, W. L.; Silva Filho, C. G.; Milioli, F. E.; Quim. Nova 2004, 27, 58.

32. Ávila, I.; Crnkovic, P. M.; Milioli, F. E.; Ouim. Nova 2006, 29, 1244

33. Milne, C. R.; Silcox, G. D.; Pershing, D. W.; Ind. Eng. Chem. Res. 1990 29, 2192.

34. Fogler, H. S.; Elementos de Engenharia das Reações Químicas, $3^{\mathrm{a}}$ ed., LTC Editora: Rio de Janeiro, 2002.

35. Dam-Johansen, K.; Ostergaard, K.; Chem. Eng. Sci. 1991, 46, 827. 